Archives de sciences sociales des religions

173 | janvier-mars 2016

Edmond Ortigues : théologie, philosophie et sciences sociales

\title{
Théologie, philosophie et sciences sociales
}

Le triangle d'Ortigues

Theology, philosophy and social sciences. The triangle of Ortigues

Teología, filosofía y ciencias sociales. El triángulo de Ortigues

Bruno Karsenti

\section{(2) OpenEdition}

\section{Journals}

Édition électronique

URL : http://journals.openedition.org/assr/27596

DOI : $10.4000 /$ assr.27596

ISSN : $1777-5825$

Éditeur

Éditions de l'EHESS

Édition imprimée

Date de publication : 1 mars 2016

Pagination : 145-156

ISBN : 978-2-7132-2516-1

ISSN : 0335-5985

\section{Référence électronique}

Bruno Karsenti, «Théologie, philosophie et sciences sociales 》, Archives de sciences sociales des religions [En ligne], 173 I janvier-mars 2016, mis en ligne le 01 mars 2019, consulté le 19 avril 2019. URL : http://journals.openedition.org/assr/27596 ; DOI : 10.4000/assr.27596 


\section{Bruno Karsenti}

\section{Théologie, philosophie et sciences sociales Le triangle d'Ortigues}

La lecture que l'on proposera ici de certains textes d'Ortigues consiste à se déplacer dans un triangle des savoirs : entre théologie, philosophie, et sciences sociales et historiques du religieux. Plus exactement, en faisant résonner dans des textes plus tardifs certains énoncés clefs du manuscrit de 1948 "Histoire et Parole de Dieu », il s'agira de dégager une certaine architecture de la pensée d'Ortigues, dont l'œuvre gravite autour de ces trois pôles. Il semble en effet que, grâce à ce manuscrit, on soit en mesure de mieux saisir les raisons pour lesquelles cette articulation doit être construite, en même temps que les problèmes qu'elle pose.

Partons du problème du rapport entre sciences sociales et théologie tel qu'il apparaît au début de la deuxième section de cette thèse de théologie. Tout sociologue, tout anthropologue ou tout historien des religions peut facilement consentir à ce qui est ici affirmé. C'est que l'ouverture à la théologie commence à devenir, non pas seulement pertinente, mais même nécessaire, dès qu'on admet qu'une vérité religieuse ne peut pas être prise comme une donnée historique extérieure sans perdre aussitôt sa valeur de vérité. Admettre qu'il y a une vérité proprement religieuse, qu'un régime de vérité se manifeste au plan religieux, et que la science sociale du religieux commence à se justifier à partir du moment où elle entreprend de restituer un tel régime, c'est forcément faire une place dans l'analyse à la vérité religieuse comme principe de jugement, et non pas comme pure donnée de fait. Le sociologue ou l'anthropologue se demandent comment la religion fait penser et agir " en vérité ", quel mode d'existence elle instaure, et donc quelle histoire elle constitue - ce que le mot d'histoire signifie dès lors qu'on la fait émerger du jugement religieux lui-même. Et c'est là que le sociologue et l'anthropologue entrent en contact avec une démarche qui, dans l'espace religieux, est déjà confrontée à ce problème. Cette démarche, c'est précisément celle du théologien. Le théologien, dans la définition qu'en donne Ortigues dans le texte de 1948, réfléchit sur "les conditions d'authenticité de l'insertion des croyants dans l'histoire du salut ». Or l'histoire du salut l'est au sens subjectif du génitif : c'est du salut qu'il y a histoire, et ce n'est pas que le salut prend place dans 
l'histoire. L'histoire est formée par le salut dont elle est l'histoire. Ou encore : la vérité religieuse n'est pas dans l'histoire, elle est formatrice d'histoire, qui est le salut même. Soulignons : cela est constitutif de la théologie, et c'est pourquoi une science sociale du religieux conséquente ne peut lui rester indifférente. En effet, cette thèse est aussi constitutive de la science sociale du religieux au sens fort de l'expression. D'où la question : est-ce que le syntagme " histoire du salut ", même si le génitif est pris au sens subjectif dans les deux cas, a bien le même sens de part et d'autre? Le problème n'est pas de séparer un sens objectif et un sens subjectif. Une science sociale du religieux qui ne veut pas réduire le religieux à un phénomène culturel quelconque, une science sociale du religieux qui veut correctement distinguer, c'est entendu, doit aller jusqu'au génitif subjectif. Mais celui-ci est-il pour autant le même que celui qui vaut pour le théologien ?

Pour y voir plus clair dans ce rapport entre sciences sociales et théologie, et en se laissant guider par la thèse d'Ortigues, il faut pourtant passer par le troisième terme, le troisième sommet du triangle : la philosophie. En effet, il est remarquable que le théologien soit ici à la fois très proche et très distant du philosophe. Dans le texte de 1948, la philosophie comparaît de deux manières. Tout d'abord, elle prend le visage de la philosophie de l'histoire. Cet aspect n'est pas jugé très intéressant. $\mathrm{Au}$ fond, on se dit qu'elle n'est pas d'un grand secours pour le théologien chrétien, et que celui-ci lui est complètement étranger. C'est que le rôle du théologien est d'élever à la conscience de lui-même le temps du salut, et de faire de l'Église un corps efficace dans cette histoire - or il ne le peut que si cette histoire se donne comme irréductible à celle des sociétés et des institutions humaines (p. 93). De ce point de vue, les philosophies de l'histoire, si imprégnées qu'elles puissent être de christianisme (et après tout, bien des auteurs ont montré à quel point elles l'étaient), ne sont pas significatives dans le cadre interrogatif où l'on se place.

Mais il y a un second visage du philosophe, beaucoup plus proche, et qui s'avère même un attracteur puissant : c'est celui qui s'interroge sur les conditions du jugement vrai, et qui met pour cela en œuvre ce qu'Ortigues appelle la "méthode réflexive ". Incontestablement, la théologie, en elle-même, a part à ce genre d'interrogation, où la pensée s'entretient avec elle-même : elle se demande à quelle condition est possible l'auditio verbi, la métanö̈a, la réponse à l'appel messianique qui crée l'Église (p. 97). Certes, la réflexion, dans ce cadre, ne renvoie pas l'esprit individuel à lui-même. Elle revient sur l'acte par lequel Dieu se fait connaître au croyant en le convertissant à lui. En d'autres termes, elle est une méthode réflexive ordonnée à la révélation de Dieu. Il n'en reste pas moins que la question que ne peut pas ne pas se poser le théologien est : quelle forme de réflexion opère dans la révélation? C'est ici que la rencontre avec le philosophe se fait la plus impérieuse, mais aussi la plus problématique : c'est que, pour le théologien, on a alors le paradoxe d'une réflexion en extériorité.

Extériorité en un double sens: au sens de son effectuation collective et cultuelle. S'approchant du philosophe dans le mouvement par lequel on s'est 
distingué des sciences sociales du religieux, voilà alors qu'on s'en éloigne pour revenir sur le premier voisinage. Le théologien, en effet, ne peut pas s'en remettre à une topique du jugement dont le philosophe aurait par avance tracé les termes et les contours pour y situer la foi en un Dieu révélé. Bien plutôt, il lui faut travailler ce que penser veut dire, dès lors qu'on se situe le mouvement même de la révélation.

Dans un texte beaucoup plus tardif, "La Révélation et le droit ${ }^{1}$ », Ortigues sera tout à fait clair : si une généralisation de l'idée de Dieu est possible, si une perspective comparative en histoire, en anthropologie ou en sociologie des religions est pensable - et donc si les sciences sociales du religieux ont bien un domaine propre - ce n'est pas en partant de Dieu comme idée partagée par une pluralité de cultures, mais de la révélation par quoi Dieu se fait connaître et vénérer. C'est là une intuition fondamentale, matricielle, comme le montre le texte de 1948 : l'opérateur de généralisation, le levier comparatif pour saisir le fait culturel de la religion, mais sans céder sur son irréductibilité à une pure donnée de fait, c'est la révélation. C'est Dieu si l'on veut, mais seulement en tant que révélé, et donc saisi du côté de ses récepteurs, des pensées et des actes par quoi cette réception s'exprime. C’est Dieu, mais seulement distingué, discriminé, entendu différenciellement dans le flux de l'expérience. C'est pourquoi il faut ramener les sciences du religieux à la sémantique des noms divins. Le mot Dieu doit être pris comme un titre à être reconnu dans ses valeurs singulières - ce qui met au premier rang de l'analyse sémantique la concentration sur le culte, par quoi toute religion s'accomplit. Dieu n'existe qu'en se révélant, ce qui signifie qu'il est celui auquel on s'adresse dans le culte. Le culte, dans ces conditions, n'est pas un apparat pratique accompagnant la croyance pour la consolider ou pour témoigner d'elle : c'en est une dimension essentielle, puisque c'est dans le culte que le rapport à Dieu se réfléchit et que les attributs de Dieu se déterminent.

Mais encore faut-il pour cela que le culte, la façon de s'adresser à Dieu correctement de sorte à entendre son message et à agir selon lui, puisse se justifier historiquement. C'est là qu'on voit comment s'articulent exactement la question historique et la question religieuse, dans le cadre de la révélation. Toute religion se donne comme circulation de signes reçus, par quoi des dieux ou des êtres surnaturels sont distingués au dehors, dans l'expérience d'une société déterminée, et par quoi cette expérience est normée d'une certaine manière. Cette normativité se confond avec le culte comme conduite adressée, où la source de la norme est reconnue dans ce qu'elle a à dire, c'est-à-dire dans sa valeur propre, distincte des autres valeurs significatives dont l'expérience peut être porteuse. Mais pour que ce message parvienne au croyant, il faut encore quelque chose de plus : il faut une origine de la relation d'adresse, il faut pouvoir se dire comment la révélation a commencé. Soulignons : cela, pour Ortigues, n’est pas spécifique à ce que nous

1. "La Révélation et le droit ", in La Révélation et le droit, Beauchesne, 2007, p. 191-202. 
désignons de façon restreinte par religion de la révélation. Dès qu'il y a du religieux, le problème de la révélation au sens large - soit le problème de l'adresse et de la reconnaissance, le problème des signes divins - est posé. Or en se posant, il soulève aussi immédiatement un problème d'histoire. Là aussi, comme pour le mot de révélation, il faut entendre le sens le plus large, celui que la généralisation par le culte, et pas par l'idée de Dieu, rend accessible. L'histoire, c'est d'abord, non pas le cadre temporel dans lequel la révélation a lieu, mais son récit, le récit du mode par lequel Dieu s'est révélé, et par là l'origine à quoi la réception actuelle des signes peut se rattacher. Il n'y a pas de culte, en ce sens, sans mythe étiologique ou sans théophanie, ce genre de mythe étant l'historicité propre à la religion comme processus cultuel - culturel si l'on veut, mais culturel seulement en tant que cultuel.

Mais la théophanie n'est pas la théologie. En faisant le détour par le texte tardif « La révélation et le droit » pour comprendre en quoi la réflexion que cible le théologien n'est pas celle que cherche à analyser le philosophe, et en l'amenant de nouveau à côtoyer l'anthropologue, le sociologue ou l'historien des religions, on bute sur une différence fondamentale. C'est que toute religion, prise au sens général qu'on vient de voir, ne comporte pas de théologie au sens strict - et que ce que la théologie produit de démarche réflexive dans la foi n'est pas explicable si l'on s'en tient à ce plan général. Il arrive quelque chose de particulier à la révélation et à l'histoire quand on se porte du côté de théologien. Plus encore, on s'aperçoit que la démarche d'Ortigues s'est élaborée dans le sens inverse. C'est en partant de ce que la théologie - et plus étroitement encore la théologie chrétienne - a à faire, que sa double incursion, dans les sciences sociales et dans la philosophie, s'est imposée d'elle-même. C'est en tout cas ce que révèle le développement de sa pensée si on s'efforce de le reconstituer à partir du texte de 1948. Citons le début de ce texte :

"Tout progrès dans l'ordre des connaissances positives, historiques, appelle un effort correspondant de réflexion pour que les acquisitions objectives rendent possible une intelligence plus avertie des réalités spirituelles» (p. 1).

Le but, ici, est théologique - rendre la théologie plus avertie, sous le coup des progrès de la connaissance historique ou sociohistorique. Mais ces progrès ne se produisent pas à l'extérieur de l'expérience du croyant. Ils sont perçus au contraire par le croyant, ajoute immédiatement Ortigues, comme de nouveaux moyens de "chercher dans le réalisme de l'histoire la réalité de la parole de Dieu » (ibid.). Ils sont donc des indices d'une articulation plus réfléchie entre histoire et révélation. Cela, le point de vue comparatif, de sociologie et d'anthropologie religieuse et d'histoire des religions, y pourvoit. Mais, sans même avoir à sortir de la religion chrétienne, y pourvoit déjà une certaine manière de prendre la réflexion théologique qui signe la spécificité du christianisme - ou du moins, du judaïsme et du christianisme dès lors qu'on les prend ensemble, dans l'unité des 
deux Testaments, et donc dans l'unité de ce que notre tradition appelle l'Écriture sainte. Voilà sur quelles bases la considération de la religion prend son envol.

Dans «La révélation et le droit », on partait donc du concept large de révélation, et on le spécifiait. Dans le texte de 1948, on part au contraire du concept spécifique. Mais même s’ils ne prennent pas le même point de départ, les deux textes se confirment. Le problème de la révélation en un sens du terme qui ne convient qu'à ce qu'on appelle les religions de la révélation, c'est que le culte et l'histoire s'y composent d'une manière très particulière. Et, au fond, la théologie émerge de ce contexte et seulement de lui, elle n'est pas suscitée par les autres modes de composition entre culte et histoire. Ce qui se révèle, et est par là reconnu dans le culte qu'on lui rend, ne vient pas prendre sens dans un mythe étiologique, il n'est pas suspendu à une histoire des origines, mais s'insère dans une alliance, et donc devient inséparable de l'itinéraire du collectif par lequel le message est reçu. On touche à ce qu'on disait en commençant : l'histoire de la révélation, c'est l'histoire du salut, au sens où c'est bien du salut qu'il y a histoire. La révélation et l'histoire communiquent à un niveau qui ne vaut que dans ce genre de religion, parce que Dieu se révèle dans l'évolution spirituelle de son peuple, successivement du peuple juif et de l'Église. Ou encore : le récit de la réception se confond avec la loi, et donne tout son contenu à la révélation. Sans doute y a-t-il toujours du mythe. Mais pour Ortigues, celui-ci se concentre alors dans la production apocalyptique, il est projeté dans le temps de la fin, et non plus retenu au temps des commencements.

C'est aussi pourquoi l'histoire positive concerne forcément le croyant en excédant toujours ce qu'elle comporte de positivité. Non parce que s'y superpose une dimension mythique. Mais au contraire parce que l'idée même d'histoire, au sens du devenir d'un collectif humain où du sens se révèle à lui-même, correspond à l'expérience proprement judéo-chrétienne de la révélation, qu'elle est ce qui vient préciser, spécifier et circonscrire la structure religieuse générique de la révélation dans ce cas singulier. L'histoire comme histoire des rapports entre Dieu et les hommes n'est pas une autre histoire que l'histoire positive qui se dégage à l'âge de la science historique. Plus exactement, il revient précisément au théologien d'articuler sa réflexion sur l'histoire du salut à cette positivité même. Comme on l'a dit, la théologie telle qu'elle se présente dans le texte de 1948 reconnaît là sa tâche majeure : insérer la communauté ecclésiale dans l'histoire du salut, lui permettre de lire et de s'orienter dans l'histoire à la lumière de son histoire.

Dans le texte de 1948, Ortigues emploie encore une autre expression pour définir cette façon théologique de poser le problème de l'histoire, d'aborder la réalité historique comme faisant partie de l'expérience des croyants rassemblés dans l'Église. Il dit que le théologien doit toujours chercher à conjuguer certains signes extérieurs et la lumière intérieure de la foi. Il semble alors que cette formulation du problème permette de situer mieux encore la réflexion théologique par rapport aux deux pôles que représentent la réflexion philosophique d'un côté, 
la connaissance sociohistorique de l'autre. Mettre en rapport certains signes extérieurs et la lumière intérieure de la foi, cela ne veut pas dire retrouver à l'extérieur la confirmation d'un sentiment intérieur. Car si c'était le cas, rien ne distinguerait la réflexion théologique d'une forme de réflexion philosophique. Sous un autre angle, on retrouve notre problème précédent, celui de la distinction entre les manifestations historiques du religieux et l'histoire du salut. Si la théologie dégage les conditions de l'insertion des actes et des paroles dans l'histoire du salut, alors elle doit s'attacher à un certain mouvement de la foi, qui n'est pas simplement d'adhésion en intériorité à une vérité posée à l'extérieur. L'histoire du christianisme, dès lors qu'elle accorde une place au discours théologique, est confrontée à un enjeu beaucoup plus radical : elle doit admettre que la transcendance de Dieu est produite dans la foi elle-même, et que les signes extérieurs, historiques, de la révélation, n'acquièrent pour le croyant leur sens que dans l'écart qui les renvoie à Dieu. La foi n'est foi que si elle passe par Dieu. C'est pourquoi la réflexion théologique ne peut être qu'analogique (p. 137). Elle est donc une réflexion qui, non seulement, passe par une extériorité, mais la maintient comme extériorité au moment même où elle opère le retour sur soi. Ortigues y insiste : il n'y a pas d'unité rétablie par la réflexion théologique, il n'y a pas de troisième terme qui viendrait subsumer le rapport entre Dieu et le fidèle. Il y a opération de synthèse en philosophie, mais pas en théologie.

Or c'est seulement si l'on analyse cette forme de réflexion décentrée, en extériorité, que l'on peut revenir sur les formes sociohistoriques dans lesquelles la foi s'est manifestée, et aborder correctement la conjonction qui peut se faire entre sciences sociales et théologie afin d'éclairer ces formes. Bref, on voit que l'effort d'Ortigues, dans le texte de 1948, est de dégager pour la théologie une voie propre, qui lui permette de se tourner vers les sciences sociales et historiques sans se trahir, mais au contraire en mettant en œuvre de façon plus lucide la méthode réflexive qui la caractérise - cette réflexion décentrée et conduite en extériorité qui se résume dans "l'analogie de la foi ", et qui nous donne accès à l'histoire comme histoire du salut telle que l'expérience chrétienne - ou judéochrétienne, mais sous réserve de ce qu'il faudrait entendre par salut du peuple dans le judaïsme - l'a introduite dans l'histoire des religions.

À partir de là, on peut s'efforcer de suivre quelques aspects de ce questionnement dans le livre de philosophie d'Ortigues, le Discours et le symbole, de 1962. Après avoir surtout évoqué le rapport entre théologie et sciences sociales du religieux, ce sera l'occasion de préciser l'autre point de la triangulation : le rapport entre théologie et philosophie.

Dans ce livre, le point de vue adopté est tout à fait différent : il est celui d'un philosophe, et plus exactement d'un philosophe qui étudie la façon dont les sciences sociales renouvellent en profondeur les conceptions philosophiques sur les conditions de formation du sens. Et pourtant, une continuité évidente relie les deux réflexions, celle du théologien et du philosophe : d'un côté comme de 
l'autre, le point d'ancrage est la parole, parole humaine et parole de Dieu, et la façon dont la pensée réflexive s'y constitue.

Or une fois qu'on a pris connaissance du texte de 1948, une intention du texte de 1962 devient plus claire et plus compréhensible. C'est qu'en se tournant vers le symbole, ou vers l'ordre symbolique sous-jacent au discours, Ortigues a cherché, du côté de l'analyse philosophique, à recueillir un aspect essentiel de ce qu'il avait ressaisi en 1948 à travers l'analogie de la foi, prise comme cette méthode réflexive propre à la pensée théologique. Pour le dire succinctement : il semble qu'il ait réfracté en philosophie cette réflexion en extériorité qui lui avait paru distinguer la réflexion théologique. Et c'est de cette manière qu'il en est venu à présenter un portrait tout à fait singulier, qui n'a pas encore été mesuré dans toutes ses implications, du paradigme structural. Par ce biais, il en est venu à construire un style d'analyse philosophique particulièrement adéquat à ce qui pourrait prétendre au statut d'une authentique philosophie des sciences sociales.

On se contentera ici de lire à la lumière du texte de 1948 quelques passages du début et de la fin du Discours et le symbole. Ce livre se présente comme une réaction, ou le diagnostic d'une réaction déjà à l'œuvre, par rapport à la philosophie moderne, celle qui s'est imposée au moins depuis Descartes, ou encore depuis le moment galiléen de mathématisation de la nature. Ce qu'Ortigues retient de ce moment moderne, c'est le dépouillement de l'individu de tous les intermédiaires qui le reliaient aux autres êtres, qu'il s'agisse des autres individus à l'intérieur de la société, ou plus largement des êtres naturels avec lesquels sa société est en rapport et qu'elle intègre à son ordre propre. Un certain ordre s'est effondré, fait de médiations qui disposaient hiérarchiquement, et selon une hiérarchie posée comme immuable, les êtres sociaux et naturels. Le moment moderne se signale à ce titre par le fait qu'on se trouve alors dans une situation de "jonction immédiate de l'individu avec l'universel ${ }^{2}$ » - formule de Feuerbach que Ortigues reprend à la première page de son livre.

Cette jonction immédiate revêt plusieurs aspects : le premier tient à l'affirmation de la conscience, prise comme principe de commencement, aussi bien de la science que de l'action. La philosophie moderne se donne alors comme recherche d'un commencement radical, dans une reprise de la pensée par elle-même, dans une pensée réfléchissante que le texte de 1948 avait justement nommé " philosophie », pour la distinguer de la réflexion théologique. C'est aussi selon Ortigues ce qu'on trouve à la base de la mathématisation de la nature, la mathématique étant le type de cette pensée opérante, où les objets ne sont rejoints que comme les résultats d'un processus de pensée effectué par le sujet connaissant. On a alors une genèse rationnelle de l'objet, depuis le sujet qui le pense. Du même coup, la philosophie de la nature se donne comme dépendante d'une philosophie du sujet, en tant que ce sujet transcende la nature. Transcendance vide, reprise dans une

2. Le discours et le symbole, Aubier, 1962, p. 10. 
conscience originaire de soi, dont le cogito cartésien offre le paradigme. Or cela emportait avec soi une certaine conception de la parole et du discours pris comme expression de cette subjectivité fondatrice. Le discours est d'abord le lieu de manifestation d'une liberté subjective, au-delà de la nature et de ses contraintes. Et, second point, la liberté est appelée à se révéler dans l'histoire, parce qu'elle se révèle d'abord dans un processus de pensée dont le sujet est le maître, pour autant qu'il suit la marche méthodique de la raison connaissante.

C'est ici que l'arrière-plan chrétien de ce diagnostic sur le moment moderne apparaît. C'est que le christianisme a accompagné et a favorisé cette construction moderne, mais qu'il l'a fait d'une manière dont la pensée moderne n'a pas pleinement conscience - et que restituer cette condition chrétienne de la modernité amène à modifier le regard que cette modernité est appelée à porter sur elle-même. Ce qui pour Ortigues sépare les classiques des modernes - les classiques voulant dire pour lui les anciens, ceux pour qui le rapport à soi et au monde ne se donne pas dans cette jonction immédiate de l'individu à l'universel, mais passe par un réseau de médiations hiérarchisées au sein d'un ordre dont l'objectivité et l'immuabilité ne font pas question - c'est un certain creusement et une certaine radicalisation de la pensée chrétienne. Dans le système où prévalait la contemplation cosmologique, en effet, la raison intervient comme un élément auquel le sujet accède, vers lequel il s'élève, qui vient sanctionner ultimement un processus de pensée qui ne la suppose pas. Pour les modernes, au contraire, la raison est ce qui se donne au départ comme puissance d'analyse et de synthèse, elle est un " infini auquel la liberté s'adosse ${ }^{3}$ ", un point de départ dont il faut manifester la fécondité interne, aussi bien dans la nature que dans l'histoire.

Or cela, dit Ortigues discrètement dans une note ${ }^{4}$, ne fait en réalité que reproduire et transposer le schéma de la théologie chrétienne. Plus précisément : de la théologie chrétienne dans sa version thomiste. Par la raison naturelle, Deus cognoscitur tanquam ignotum. Autrement dit, c'est en dépassant le monde naturel, connu à travers les êtres sensibles, que l'on s'élève à la connaissance de Dieu, laquelle est toujours en dernière analyse celle d'un Dieu caché. Ce Dieu caché se manifeste dans l'histoire par sa parole, et la théologie, en s'attachant à cette parole, accède à la manifestation historique de celui qui reste caché tout en parlant aux hommes. Au Dieu créateur caché, la théologie ne renvoie qu'en passant par la parole divine, révélation de Dieu, Dieu révélé dans l'histoire humaine. Pour Ortigues, il est clair alors que cette distinction entre Dieu créateur caché et Dieu révélé dans sa parole, enferme déjà in nuce la coupure moderne - une coupure que la modernité ne fera que redécouvrir avec les armes de la science et de la philosophie. Pourquoi cela ? Parce qu'on voit déjà que le caché est mis en retrait de l'esprit connaissant, sous l'espèce du Dieu créateur, et cela précisément pour

3. Le Discours et le symbole, op. cit., p. 11.

4. Ibid. 
libérer la pénétration rationnelle de la parole divine comme révélation dans l'histoire. On a donc, dès la dogmatique théologique du XIII ${ }^{\mathrm{e}}$ siècle, le schéma qui importe à une philosophie de la conscience, et qui devait ultimement trouver sa mise en œuvre aussi bien dans la mathématisation de la nature que dans la philosophie de l'histoire. L'esprit pénètre le monde en le reconstruisant à travers une parole dont le déploiement est coextensif du mouvement de la raison. L'élément mystique - l'expression est d'Ortigues ${ }^{5}$ - que les Grecs posaient comme un horizon ou un terme ultime, est rejeté en arrière, résorbé dans la création, qui peut ainsi se donner aussi bien comme point d'origine de la subjectivité elle-même, transcendance vide qui surplombe la société historique comme elle surplombe la nature. Lorsqu'on assiste, au XVII siècle, à un renversement du couple Dieu cachél Dieu révélé, comme c'est le cas chez Pascal - le Dieu caché devenant le Dieu de l'histoire, et le Dieu créateur un Dieu sans mystère, le Dieu du déisme ${ }^{6}$-, il faut y voir un retour de ce qu'on avait mis à distance - et Ortigues n'hésite pas alors à parler à ce propos de résurgence de l'hellénisme ${ }^{7}$. Cela ne veut évidemment pas dire que le christianisme se perde dans cette inversion. Mais cela veut dire du moins que se trouble l'affinité qu'il y a entre la théologie chrétienne et la philosophie de la conscience. On sait que, précisément, la séparation entre philosophie et théologie en a découlé. Mais ce qu'il faut y voir, c'est moins une émancipation de la philosophie de la dogmatique théologique, qu'un obscurcissement du socle théologique qui a permis à la conscience de se séparer du dispositif cosmologique, et qui a donné son impulsion à notre pensée de la nature et de l'histoire. La philosophie du sujet est alors philosophie de l'action humaine, action historique, inscription de la raison dans un monde que l'homme peut connaître dans la mesure où il peut y agir réellement, c'est-à-dire librement. De même, dans cette perspective, ce qu'on appelle le discours, le fait que l'homme parle et sa production de parole, vient se soumettre à la norme d'une vérité fondée en raison, c'est-à-dire inscriptible dans le procès de la raison par lequel la subjectivité se manifeste et se déploie. La parole et l'histoire sortent de la bouche et des mains d'un sujet qui se tient seul, affranchi de son appartenance à un ordre qui le précède et l'assigne à parler comme il parle et à agir comme il agit.

Or cette jonction de l'homme et de l'universel ne va pas sans écueils, et la modernité, c'est aussi et peut-être surtout l'histoire de ces écueils, de cette opacité reconnue au donné historique et social, de cette épaisseur du discours comme quelque chose qui échappe aux constructions subjectives du sens. Cette résistance éprouvée, on peut dire qu'elle est à l'origine d'une autre bifurcation, typiquement moderne cette fois : celle entre philosophie et sciences sociales, ces dernières

5. Le Discours et le symbole, p. 11.

6. Sur ce point, évoqué subrepticement dans le Discours et le symbole, voir surtout "Que veut dire mystique? ", in Le Temps de la parole, Presses universitaires de Rennes, p. 95.

7. Le Discours et le symbole, p. 12. 
prenant leur essor dans la reconnaissance d'une opacité constitutive des « choses » sociales et historiques, ces choses dont Durkheim nous dit bien comment il faut les prendre : non immédiatement " compénétrables à l'intelligence ${ }^{8}$ ", analysables à condition qu'on leur accorde cette dimension d'extériorité que l'on s'efforce de pénétrer, mais non pas d'abolir.

Ce n'est pourtant pas en suivant cette bifurcation-là qu'Ortigues conduit sa réflexion. Ce qu'il suggère, c'est que, d'une certaine manière, cette résistance éprouvée pouvait déjà se lire dans le renversement pascalien de la distinction thomiste entre Dieu caché et Dieu révélé. La conséquence est alors la suivante : c'est qu'il y a bien une histoire de la réflexion théologique qui ne coïncide pas avec sa transposition qui s'est accomplie dans la philosophie moderne. Si Dieu est caché dans l'histoire, et révélé au croyant, c'est qu'on ne peut pas rabattre la révélation de Dieu sur le développement du sens dans le donné historique. On retrouve ainsi un paradoxe proprement chrétien, qui n'est rien d'autre, si l'on reprend la dénomination du texte de 1948, que le paradoxe de l'analogie de la foi, ce paradoxe qui veut que lumière de la foi, dans le moment même où elle reconnaît les signes historiques de la révélation, sorte d'elle-même pour prendre sa source en Dieu. Dans le texte de 1948, Ortigues avait affirmé, on l'a vu, que l'analogie de la foi rendait possible la "méthode réflexive» en théologie. Dans le livre de 1962, il n'a pas recours à l'analogie de la foi, mais à une situation de crise de la méthode réflexive en philosophie. Il reste que cette crise porte la trace d'un problème proprement chrétien, celui d'avoir fourni son schème à l'humanisme athée - on peut reprendre ici la dénomination d'Henri de Lubac - et, dans le même temps, de ne pas cesser de manifester les limites de la transposition strictement philosophique de ce schème. À cet égard, il serait tout à fait légitime de chercher dans l'histoire de la théologie et des transformations de l'analogie de la foi, c'est-à-dire de son mode de réflexion caractéristique, de quoi nous éclairer sur ce que la philosophie moderne comporte d'illusions lorsqu'elle traite de l'histoire et des sociétés humaines d'un point de vue subjectiviste.

Une autre méthode, plus directe, est cependant possible, et il semble que ce soit celle qu'a suivie Ortigues : c'est de prendre appui sur la bifurcation, ou du moins la tension épistémique évoquée plus haut, celle entre sciences sociales et philosophie, afin de déterminer en quoi elle a pu renouveler ce qu'il convient d'entendre par "méthode réflexive ", du point de vue même de la philosophie. Ou encore, on peut s'efforcer de mesurer l'impact sur les conceptions philosophiques de la construction du sens dans le discours produit par les enquêtes des

8. Les règles de la méthode sociologique, préface à la seconde édition, Presses universitaires de France, 1987, p. XIII. Si je puis me permettre ici une notation subjective - autorisée peutêtre par le fait que l'un des grandes vertus de l'œuvre d'Ortigues est de permettre à son lecteur de mieux se situer lui-même : c'est dans cette voie que je me suis personnellement engagé, $c f$. D’une philosophie à l'autre. Les sciences sociales et la politique des modernes, Gallimard, 2013. 
linguistes, des sociologues, des historiens, des psychologues. Cela, c'est bien la trame choisie dans le Discours et le symbole pour renouveler la philosophie moderne, pour refuser l'abolition des médiations par quoi elle a pris son essor, et la relancer sur un autre plan, où il devient possible de recomposer les relations indirectes du sujet et du sens. En bien des points - qu'on pense par exemple à la reprise du De Trinitate et du cogito augustinien à la fin du livre ${ }^{9}$ - cette démarche philosophique conserve plus qu'un écho de la préoccupation théologique qui l'avait amorcée. Mais justement, il me semble qu'en relisant le Discours et le symbole à la lumière du texte de 1948, ce qu'on réalise, c'est que cette amorce pointait vers une ambiguïté constitutive de la théologie chrétienne, qui est à la fois de servir de source inconsciente de la philosophie du sujet, et d'en faire ressortir les limites en termes de connaissance. Ce qu'il faudrait ici suivre de près, pour éclairer cette ambiguïté, c'est le passage de l'analogie de la foi comme forme de réflexion théologique, à la symbolisation comme condition du discours et de la parole qu'Ortigues redécouvre en empruntant la voie de l'analyse philosophique. Ou encore, c'est le passage de l'histoire du salut, et donc de la production d'historicité constitutive de la pensée théologique, à l'analyse de la temporalité propre à la parole humaine que le Discours et le symbole parvient à conduire, précisément en distinguant rapports symboliques et rapports de signification et en montrant comment ils s'articulent. Telles sont les pistes de travail sur l'œuvre d'Ortigues que la lecture du texte de 1948 permet d'entrevoir.

\section{Bruno KARSENTI \\ Laboratoire interdisciplinaire d'études sur les réflexivités \\ Lier, EHESS \\ karsenti@ehess.fr}

\section{Théologie, philosophie et sciences sociales. Le triangle d'Ortigues}

L'objet de cette étude est de confronter la thèse de théologie d'Ortigues de 1948 à certaines propositions développées dans le Discours et le symbole quelques années plus tard, en 1962. L'ensemble de sa pensée s'articule en effet autour de trois pôles: l'anthropologie et les sciences historiques du religieux, la réflexion théologique, et la philosophie comme discipline du jugement. Or c'est la genèse de ce trièdre qui devient compréhensible à partir de ce texte précoce. Sur la base d'une définition de la théologie comme démarche réflexive de la foi en la parole de Dieu et inscription du croyant dans le temps $d u$ salut, Ortigues parvient à fournir aux sciences sociales du religieux un cadre épistémologique précis, et à conférer un sens inédit à l'orientation de la philosophie moderne.

Mots-clés : théologie, histoire, salut, philosophie, réflexivité, foi.

9. Le Discours et le symbole, p. 208 sq. 


\section{Theology, philosophy and social sciences. The triangle of Ortigues}

The object of this study is to confront the thesis in Theology of Ortigues in 1948 with some propositions developed in le Discours et le symbole a few years later, in 1962. Indeed, his thought a sa whole is generally articulated around three poles: anthropology and historical science of the religious; theological reflection; and philosophy as a discipline of judgment. Or it is the genesis of this tribedron that starts making sense from this early text. On the basis of a definition of Theology as the reflexive endeavor of the faith in the word of God and of the inscription of the believer in the time of salvation, Ortigues manages to provide the Social Sciences of the religious with a precise epistemological frame, and to confer an unprecedented meaning to the orientation of modern Philosophy.

Key words: theology, history, salvation, philosophy, reflexivity, faith.

\section{Teología, filosofía y ciencias sociales. El triángulo de Ortigues}

El objeto de este estudio es confrontar la tesis de teología de Ortigues de 1948 con ciertas proposiciones desarrolladas en le Discours et le symbole algunos años más tarde, en 1962. El conjunto de su pensamiento se articula en efecto alrededor de tres polos: la antropología y las ciencias históricas de lo religioso, la reflexión teológica, y la filosofía como disciplina de juicio. Ahora bien, es la génesis de este triedro lo que se vuelve comprensible a partir de este texto precoz. Sobre la base de una definición de la teología como camino reflexivo de la fe en la palabra de Dios y como la inscripción del creyente en el tiempo de la salvación, Ortigues necesita dotar a las ciencias sociales de lo religioso de un marco epistemológico preciso, y conferir un sentido inédito a la orientación de la filosofía moderna.

Palabras clave: teología, historia, salvación filosofía, reflexividad, fe. 